\title{
Estimación del cambio climático en la cuenca coata mediante el modelo GISS-E2-R y ver sus efectos sobre la alpaca en Puno
}

Cruz Paredes Yobana Elsa ${ }^{1 *}$, Castro Sarco Diana Gloria ${ }^{2}$, Arivilca Dominguez Javier Gustavo ${ }^{3}$

\section{Resumen}

El cambio climático está afectando a diversas regiones del mundo y el Altiplano puneño no es excepción, debido a que es considerado como una de las zonas más vulnerables ante estos cambios. Por tal motivo, el objetivo de la investigación es estimar las variables climáticas de la cuenca Coata mediante el modelo GISS-E2-R e identificar sus efectos en la crianza de alpacas en el departamento de Puno. Para ello se manejaron datos de SENAMHI y WORLDCLIM (WC) del periodo de 1960-1990, en (temperatura máxima, mínima y precipitación) de las estaciones meteorológicas Cabanillas, Juliaca, Lampa y Pampa, como la proyección climática futura estimada al 2050. Resultando, la temperatura aumentada en la zona de Lampa $3^{\circ} \mathrm{C}$ y disminuida en la zona de Cabanillas en $-7.0^{\circ} \mathrm{C}$. Con respecto a la precipitación se mostraron deficiencias mayormente en la zona de Lampa, también algunas estimaciones en la sierra de Puno, entre $-10 \%$ y $-20 \%$. Y en la selva norte y central con un $10 \%$. Por tanto, se concluye que en la costa norte y selva sur se darán los incrementos más importantes con un $-10 \%$ a $+20 \%$ según estudios realizados, y la disminución de la temperatura estima la presencia de nieve y bajas temperaturas lo que posibilita mayor cantidad de muertes de alpacas, por lo que, es recomendable proteger y evitar su exposición al friaje ayudando a su adaptabilidad a nuevas condiciones climáticas.

Palabra clave: Cambio climático, Cuenca coata, precipitación, temperaturas.

\begin{abstract}
Climate change is affecting several regions of the world and the Puno highlands is no exception, because it is considered as one of the areas most vulnerable to these changes. Therefore, the objective of the research is to estimate the climatic variables of the Coata basin using the GISS-E2-R model and to identify its effects on the breeding of alpacas in the departamento of Puno. Data from SENAMHI and WORLDCLIM (WC) from the period 19601990 were used in (maximum temperature, minimum and precipitation) of the Cabanillas, Julica, Lampa and Pampauta meteorological stations. As the projected future climate predicted to 2050, validating the data by linear trend analysis mentioned (Andersen-Jensen, 2001). As the temperature will increase in the Lampa area $3{ }^{\circ} \mathrm{C}$ and decrease in the Cabanillas area at $7.0^{\circ} \mathrm{C}$ with respect to precipitation, deficiencies were mostly observed in the Lampa area, also some estimates in the Sierra de Puno, between $-10 \%$ and $-20 \%$. And in the north and central jungle with $-10 \%$. Therefore, it is concluded that in the north coast and southern jungle, the most important increases will occur with $10 \%$ to $+20 \%$ according to studies carried out by (Lujano et al., 2016), and the decrease in temperature estimates the presence Of snow and low temperatures which it is possible that there is a greater quantity of alpaca deaths, which is recommendable to protect and to avoid its exposure to the friaje helping its adaptability to new climatic conditions
\end{abstract}

Keyword: Climate change, Coata watershed, temperatures, precipitation.

\footnotetext{
* Autor de correspondencia: Cruz Paredes Yobana Elsa

Km. 19 Carretera Central, Ñaña, Lima

Tel.: +51-960-080-205

E-mail:, yobanacruz88@gmail.com, dianacastro@upeu.edu.pe, javierarivilca@upeu.edu.pe
} 


\section{Introducción}

El cambio Climático es definitivamente uno de los temas más relevantes de la agenda ambiental internacional actual. Lo que se evidencia que en los últimos años el comportamiento climático se presenta en el Perú, con una serie de anomalías que afecta a la agricultura, ganadería y el desarrollo del país. En la campaña que fue el 2007-2008, se presentaron irregularidades de lluvias, bajas temperaturas, nevadas y granizadas provocaron daños y pérdidas entre los agricultores y alpaqueros de las zonas altoandinos. (Mori Kuriyama \& Zafra Pozo, 2008). Estudios indican que se pueden esperar cambios en la frecuencia e intensidad de ondas de calor, sequías e inundaciones, en diversas regiones del mundo en respuesta al cambio climático global (IPCC, 2007).

En la realidad andina Peruana existen incuestionables evidencias de severos impactos de los extremos del clima que están ocurriendo o que pueden ocurrir en la región de Puno, por eso es de crucial importancia invertir en investigaciones para evaluar con mayor certeza los cambios del clima. Desde una perspectiva regional o cronológica la climatología nos ayudará a contribuir a comprender y explicar los efectos en el medio de supervivencia de las alpacas, y al resto de procesos de intercambio energético que ocurren sobre el planeta viendo si existe un calentamiento o un enfriamiento. Debido a su altitud y orografía están expuestas a factores climáticos extremos, estos interactúan entre sí creando situaciones climáticas críticas que afectan a la producción de alimentos, producción ganadera.

La región del Altiplano, contiene una alta producción de fibra de alpaca, que cuenta con un $55 \%$ por ciento de la población mundial de esta especie. Lo que su principal fuente de ingresos de los pobladores en esta zona proviene de las actividades ganadera y agrícola. La zona rural alto andino constituida como la zona alpaquera, cuya actividad principal aparte de la producción de la Quinua, es la producción de camélidos domésticos que involucran a todas las provincias (Crispin, 2008). También es considerado como el tercer departamento más pobre del Perú que depende principalmente de la actividad agropecuaria aportando a la economía del departamento con un $68.3 \%$, así mismo es uno de los tres principales productores del cultivo de papa y de alpacas a nivel nacional (INEI, 2007).

Según la revista Sol Alpaca menciona, que la alpaca es autóctona de las alturas peruana. Existen un estimado de cuatro millones de alpacas de Sudamérica, acerca del 95\% habitan en la región central de Perú. Se desarrollan de manera adecuada en alturas que oscilan entre los 3000 y más de 4500 metros (cerca de 10,000 a 15,000 pies, sobre el nivel del mar, en temperaturas que varían de $-20^{\circ} \mathrm{C}$ a $30^{\circ} \mathrm{C}$ centígrados en un mismo dia. Lo que actualmente en el altiplano se están muriendo debido a la variación de temperatura y las ocurrencias de 
precipitación que llegaron a bajas temperaturas registradas 17 de agosto de 2015. según varias regiones de Perú provocando la mortalidad de más de 180 mil camélidos, donde la temperatura descendió a 20.6 grados bajo cero sepultando así los forrajes.

Por lo que es alta la vulnerabilidad si, eventos climáticos extremos sucedieran, por eso surge la necesidad y motivación de realizar estudios de investigación que puedan ser mostrados a los órganos correspondientes, con el fin de tomar medidas preventivas y de adaptación. La finalidad de este estudio es estimar a largo plazo las condiciones atmosféricas de temperaturas y precipitación, realizado en tres partes, la primera parte consistió en la selección de un modelo climático como ACCESS 1.0 y GISS-E2-R que involucran la temperaturas y precipitación para unas estimaciones al 2050 de la cuenca Coata. Donde los datos de SENAMHI son comparados con los datos descargados de WORD CLIM.A través de validación del clima actual basados en las observaciones, los datos obtenidos fueron de las estaciones de Juliaca, Pampahuta, Cabanillas y Lampa. Finalmente se realizó la verificación de los efectos que podría ocasionar en la crianza de las alpacas del departamento de Puno.

\section{Materiales y Métodos Zona de estudio}

El área de estudio de la cuenca Coata está ubicado entre las coordenadas UTM (WGS84, zona 19 L); Esté: 282.907 - 401.525, Norte: 8’328.509 - 8’239,696 con altitudes entre 5.300 - 3.800 m.s.n.m. Se caracterizada como un sistema de cuenca endorreica, la superficie total es de 4,908.44 Km2 aproximadamente. Por otro lado, la región sierra se caracteriza por veranos lluviosos que comprende del 21 de diciembre, el otoño inicia el 19 marzo al 20 junio donde las temperaturas descienden al máximo, con un invierno frío- ventoso que inicia el 21 de junio a 22 de septiembre y primavera 23 de septiembre a 21 de diciembre (SENAMHI, 2016).

i. Base de datos

Se obtuvo información de SENAMHI - WORLDCLIM del periodo 1960 -1990, a una resolución de 30s ( 1 Km). Así mismo, se utilizó información meteorológica multi mensual (temperatura máxima, mínima y precipitación) de las estaciones de Lampa 352947.53 E, 8266710.09 O, Cabanillas 355704.37 E, 8270532.61 O, Juliaca 370412.52 E, 8292182.47 O y Pampa Uta 320230.47 E, 8287490.58 O.

\section{ii. Validación}

La línea de tendencia es la que permite en la actualidad aproximarse a las proyecciones del clima futuro y atribuir las posibles causas, en ese sentido los modelos climáticos de circulación CMIP5 se consideró el siguiente modelo GISSE2-R. Dentro de las cuales la información de datos para las proyecciones futuras corresponde a RCP 45 - 30s para el año 
2050 que es el escenario e intermedio de emisiones de gases de efecto invernadero. (Van Vuuren et al., 2011).

Asímismo, se realizó la validación de datos mediante análisis de tendencia lineal, para los datos históricos de SENAMHI y WC. Donde R2 fue clasificado bajo los criterios de muy bueno $>0.95$, bueno de $0.85-0.95$, satisfactorio de $0.65-0.85$ e insatisfactorio $<0.65$ (Andersen, Refsgaardy Jensen, 2001), más acertado los pronósticos, lo que implica una mayor correlación.

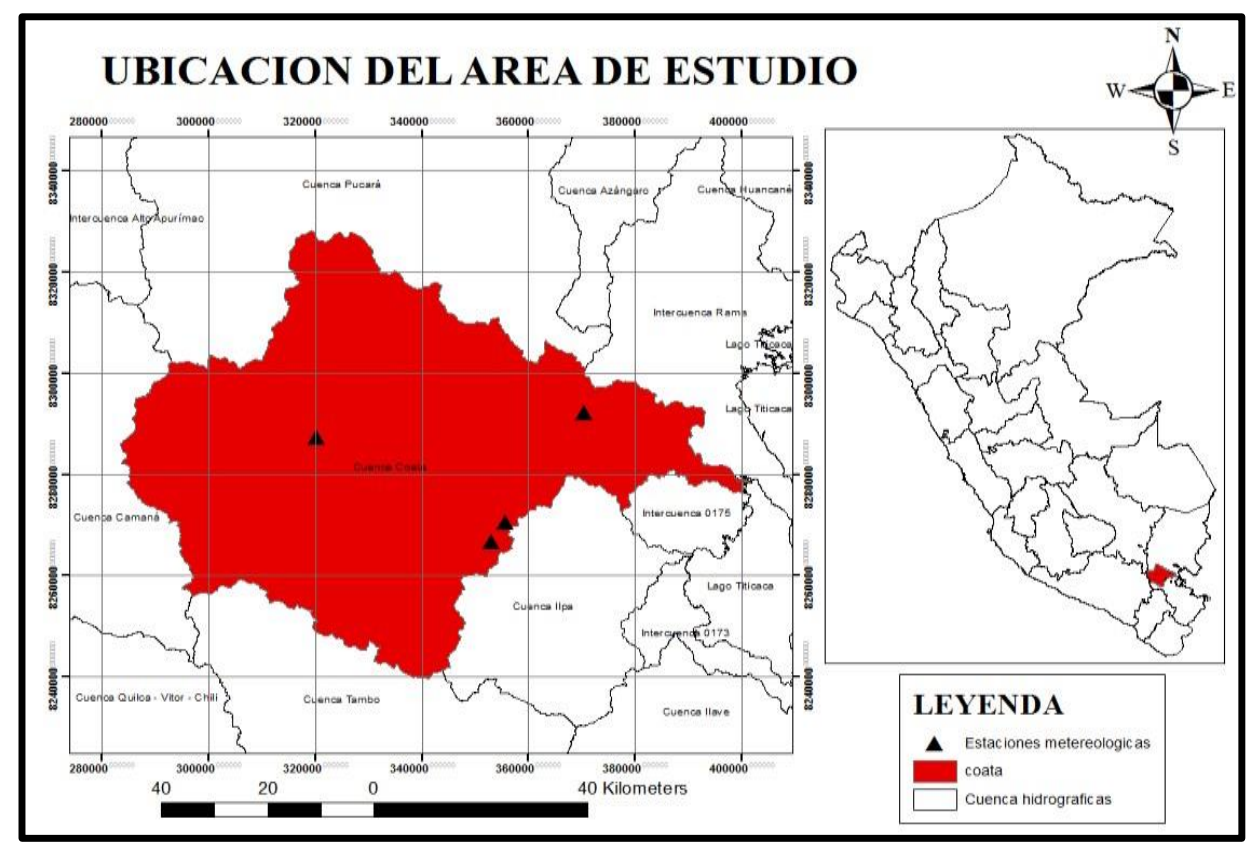

Figura 1. Mapa de ubicación de la zona de estudio.

\section{Resultados y Discusiones}

Resultados de validación de datos mediante tendencia lineal resulta. 


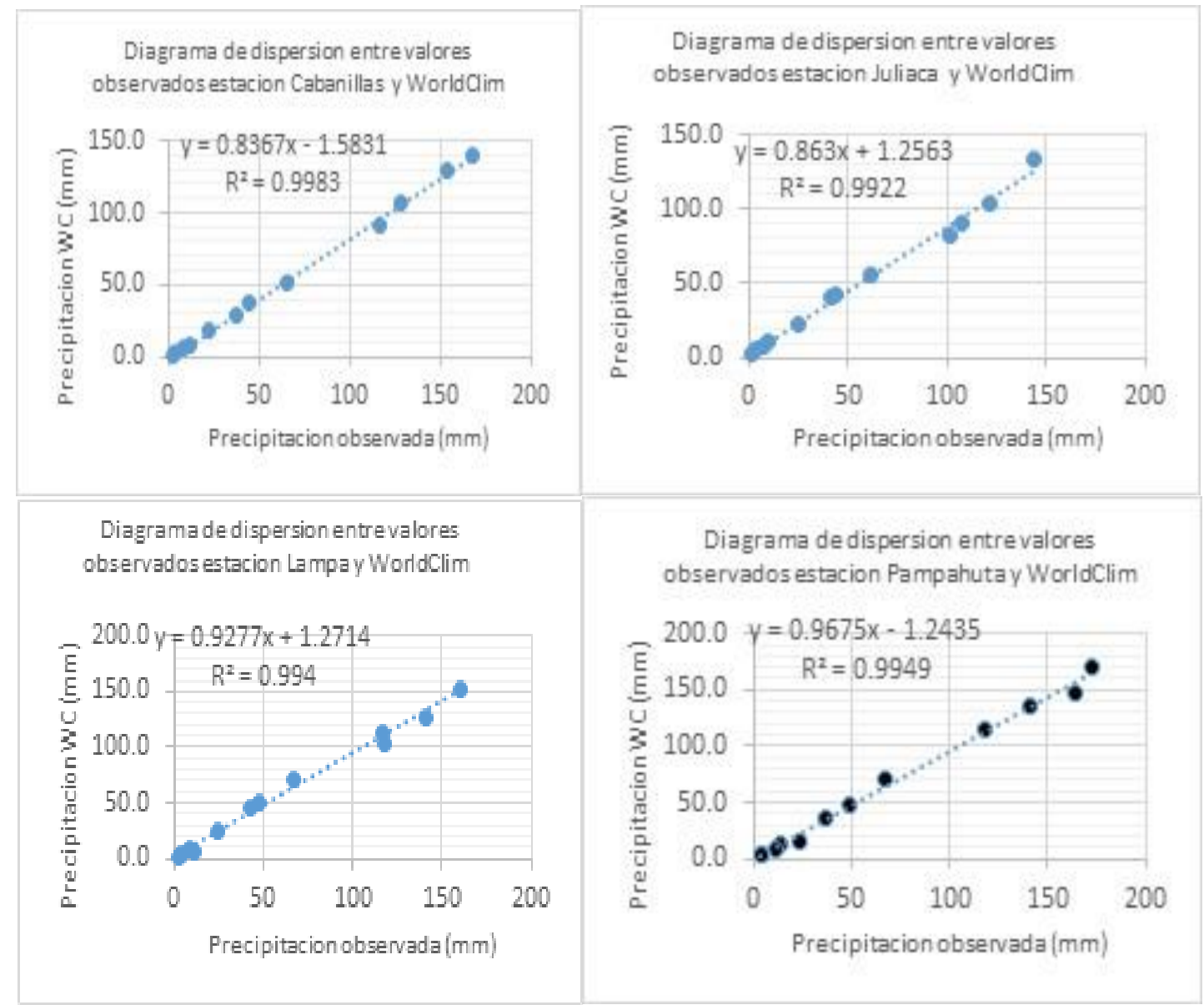

Figura 2. Precipitaciones proyectadas en las precipitaciones.

La información de WC es válida en las tendencias de Cabanillas, Juliaca, Lampa y Pampa Uta de la precipitación, debido a que presenta un coeficiente de determinación igual a 0.99, el cual es corroborado según el criterio de Andersen et al. (2001). 
i. Temperatura máxima

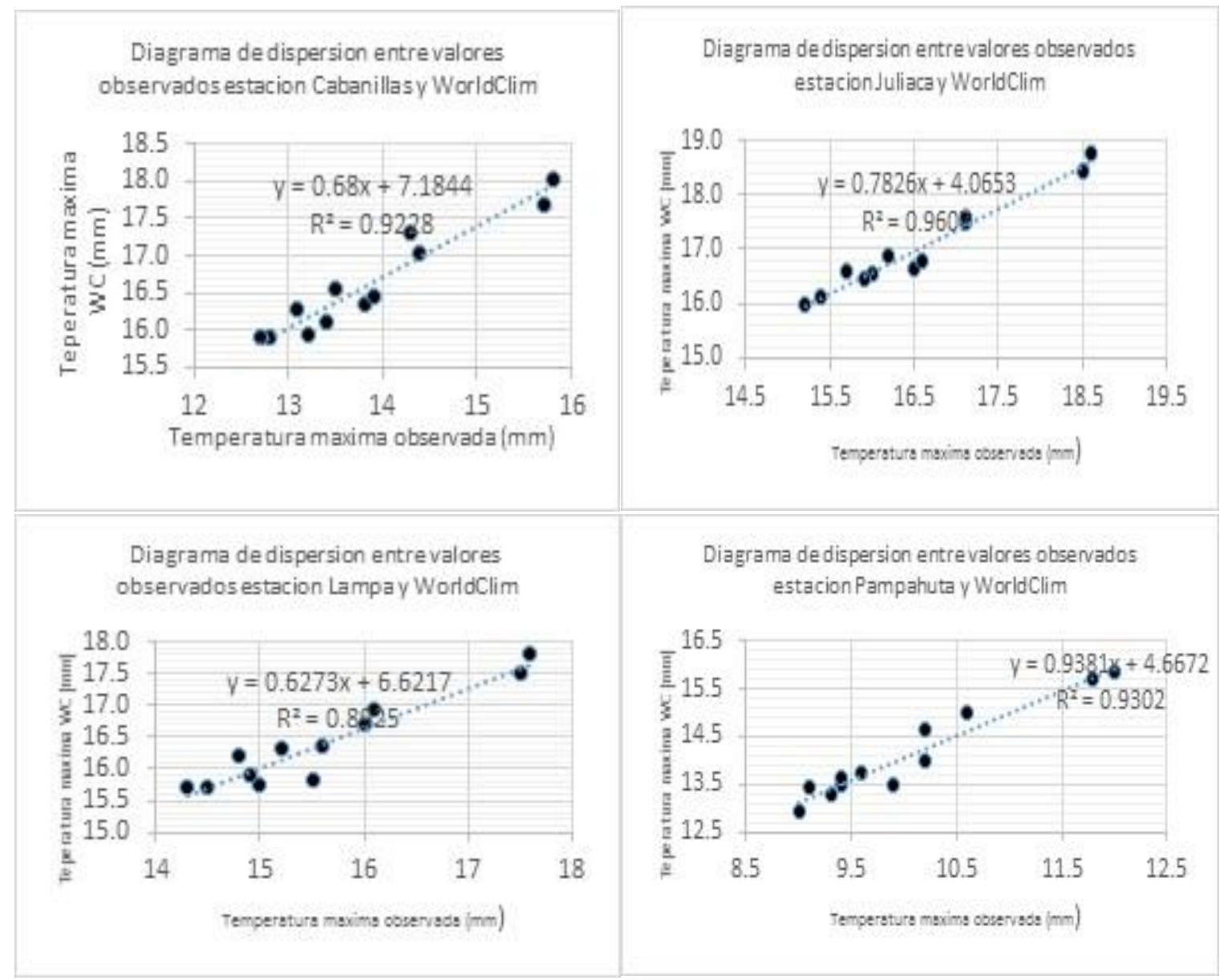

Figura 3. Diagrama de dispersión de valores observados de la temperatura máxima.

La información de WC es válida, debido a que presenta un coeficiente de determinación igual a 0.99, como en Cabanillas resulta en un 0.92, en Juliaca 0.96, Lampa en 0.88 y en Pampahuta en 0.93, el cual es corroborado según el criterio de Andersen et al. (2001). Sin embargo, lampa no tiene tan buena relación ya que es menor de 0.99, pero es Bueno. 
ii. Temperatura mínima

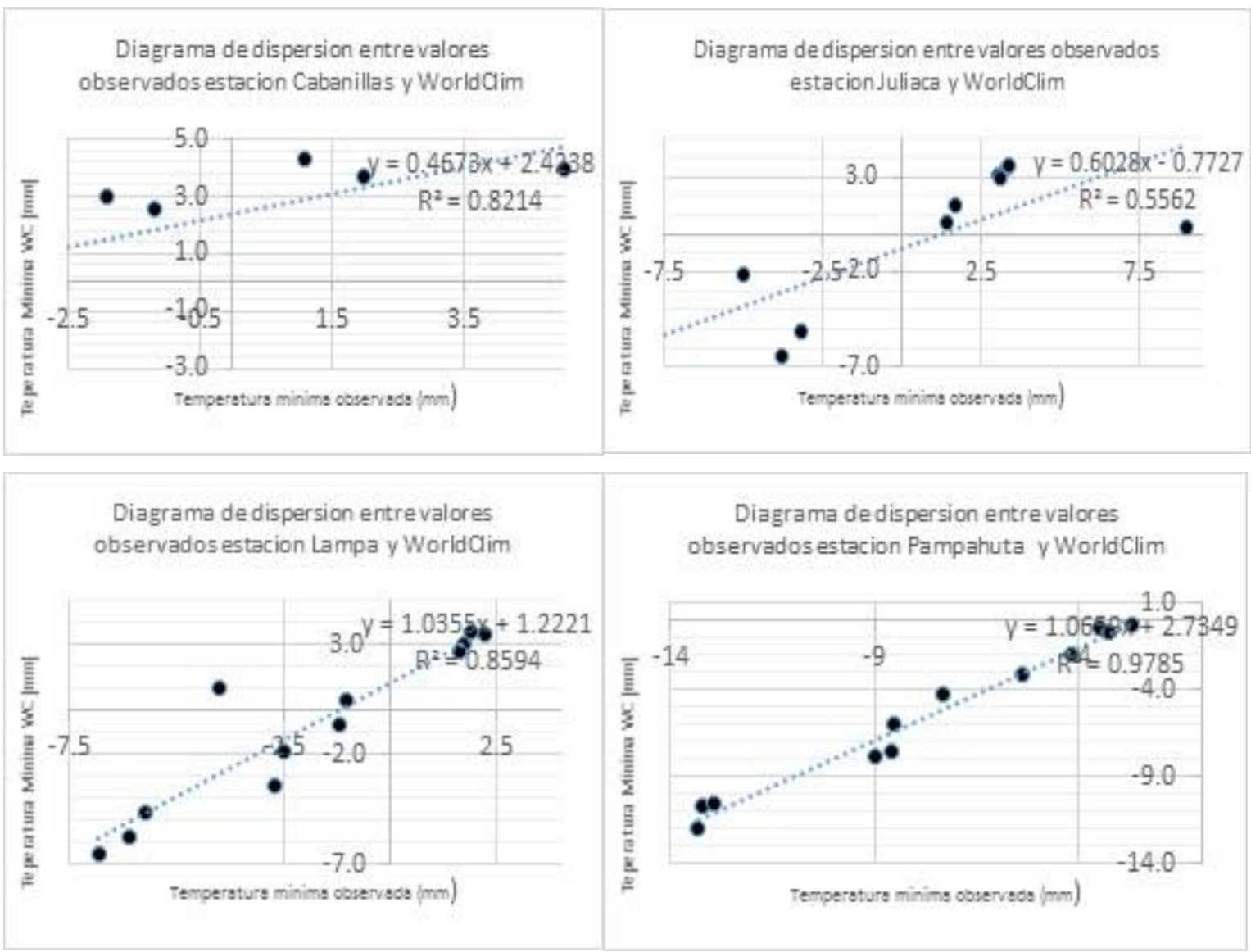

Figura 4. Diagrama de dispersión de valores observados de temperaturas mínimas.

La información de WC es válida, debido a que presenta un coeficiente de determinación igual a 0.99, el cual es corroborado según el criterio de Andersen et al. (2001). Por otro lado, lampa no tiene buena relación, mientras Juliaca es necesario realizar un diagrama de dispersión de media móvil debido a que $\mathrm{R}$ es 0.5 lo que indica en que los datos pronosticados no son tan reales.

iii. Proyección al 2050

Según el estudio realizado se evidencia variaciones de cambio climático en el régimen de temperaturas y precipitaciones para los escenarios RCP45 y horizonte 2041-2050, por el método GISS-E2-R para el 2050 en la cuenca coata, la temperatura mínima incrementará entre 0.2 y $3.8^{\circ} \mathrm{C}$, en especial en Cabanillas y lampa. Y la temperatura máxima incrementará entre 0.7 y $2.3{ }^{\circ} \mathrm{C}$. Con respecto a las precipitaciones, en el 2050 las precipitaciones anuales mostrarían deficiencias mayormente en lampa con $-91.3 \mathrm{~mm}$ y en Pampahuta incrementará en un 122 mm; según estudios realizados por (Lujano, et al., 2016) en la cuenca Ramis estiman un incremento de precipitaciones en $+8.5 \%$ en la parte norte y la zona sur las precipitaciones 
disminuirán en $(-30 \%)$. Por lo que se coincide en la cuenca coata la parte norte tiene incrementos mientras que la parte sur las precipitaciones disminuyen.

\begin{tabular}{|c|c|c|c|c|c|c|c|c|c|c|c|c|c|}
\hline \multirow[b]{2}{*}{$\mathrm{N}^{\circ}$} & \multirow[b]{2}{*}{ Meses } & \multicolumn{2}{|c|}{$\begin{array}{l}\text { Temperatura } \\
\text { diferencia }\end{array}$} & \multicolumn{2}{|c|}{ máxima } & \multicolumn{2}{|c|}{$\begin{array}{l}\text { Temperatura } \\
\text { diferencia }\end{array}$} & \multicolumn{2}{|c|}{ Mínima } & \multicolumn{4}{|c|}{ Precipitación diferencia } \\
\hline & & 营 & 㠀 & 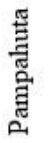 & 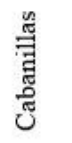 & 尝 & 莺 & 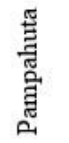 & 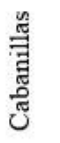 & 营 & 㥕 & 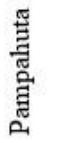 & 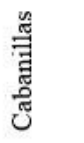 \\
\hline 1 & Enero & 2.7 & 2.6 & 1.1 & 2.1 & 1.3 & 2.8 & 1.6 & 0.9 & -24.1 & 11.4 & -0.7 & 0.2 \\
\hline 2 & Febrero & 2.5 & 2.2 & 0.7 & 2 & 1.5 & 2.5 & 2 & 0.9 & -23.4 & 17.0 & 7.8 & -3.4 \\
\hline 3 & Marzo & 2.2 & 2.4 & 0.8 & 2.1 & 1.7 & 3 & 1.8 & 1.1 & -6.5 & 15.8 & 7.1 & 1.3 \\
\hline 4 & Abril & 2.1 & 2.2 & 0.7 & 1.8 & 2.5 & 4 & -3.9 & 0.9 & -5.1 & 10.9 & 2.6 & 2.8 \\
\hline 5 & Mayo & 1.9 & 2 & 0.7 & 1.7 & 11.4 & 6.7 & 4.2 & 1.8 & 3.6 & 0.0 & 3.8 & 2.5 \\
\hline 6 & Junio & 2.2 & 2.2 & 1.1 & 1.7 & 3.6 & 11.5 & 3 & -7 & 0.8 & 0.2 & 0.6 & 2.4 \\
\hline 7 & Julio & 1.8 & 1.9 & 0.6 & 1.3 & 3.4 & 6.4 & 3.6 & 0.7 & 0.5 & 0.2 & 2.0 & -0.7 \\
\hline 8 & Agosto & 2.5 & 2.7 & 1.2 & 2.2 & 4.7 & 5.2 & 10.5 & 0.8 & -1.8 & 3.2 & 1.1 & -0.4 \\
\hline 9 & Septiembre & 2.2 & 2.3 & 0.5 & 1.9 & 8.9 & 4.9 & 3.4 & 0.2 & 1.3 & 6.8 & 8.8 & 6.0 \\
\hline 10 & Octubre & 3 & 2.8 & 0.6 & 2.4 & 2.7 & 4 & 2.3 & 0.3 & -0.5 & 11.5 & 8.9 & 8.7 \\
\hline 11 & Noviembre & 2.3 & 2.3 & 0.2 & 2 & 1.6 & 3.4 & 1.7 & 0.2 & -10.6 & 18.6 & 8.0 & 8.8 \\
\hline 12 & Diciembre & 2.2 & 2.1 & 0 & 1.5 & 1.8 & 3.1 & 4 & 1 & -25.5 & 26.5 & 13.0 & 2.7 \\
\hline $\begin{array}{l}\text { Pro } \\
\text { prec }\end{array}$ & $\begin{array}{l}\text { edio Anual } \mathrm{T}^{\circ} \mathrm{y} \\
\text { tacion sumatoria }\end{array}$ & 2.3 & 2.3 & 0.7 & 1.9 & 3.8 & 4.8 & 2.9 & 0.2 & -91.3 & 122 & 63.1 & 30.9 \\
\hline
\end{tabular}

Este pronóstico indica la diferencias de la temperatura máxima en el mes de octubre incrementará hasta $3^{\circ} \mathrm{C}$, perteneciente a la estación de Lampa, zona de mayor influencia, además lugar donde se da mayor crianza de alpacas y también influirá la temperatura mínima donde reducirá hasta $-7.0^{\circ} \mathrm{C}$, en el mes de junio zona de mayor influencia Cabanillas, este pronóstico nos ayuda a predecir la condición de vida de la alpacas, donde (Neitzke \& Alten, 1997) indica que viven entre 20-25 años, pero este periodo de vida será reducido por la afectación del clima, el equilibrio ecológico en de su hábitat es sumamente frágil, y por lo tanto su manejo productivo será difícil, también la alimentación será afectado teniendo pasto seco. Por otro lado, puede resultar una pérdida de estado corporal de la hembra lo cual puede afectar su gestación, e inducir una reducción de la producción de leche que afectará a la cría (FAO, 2005).

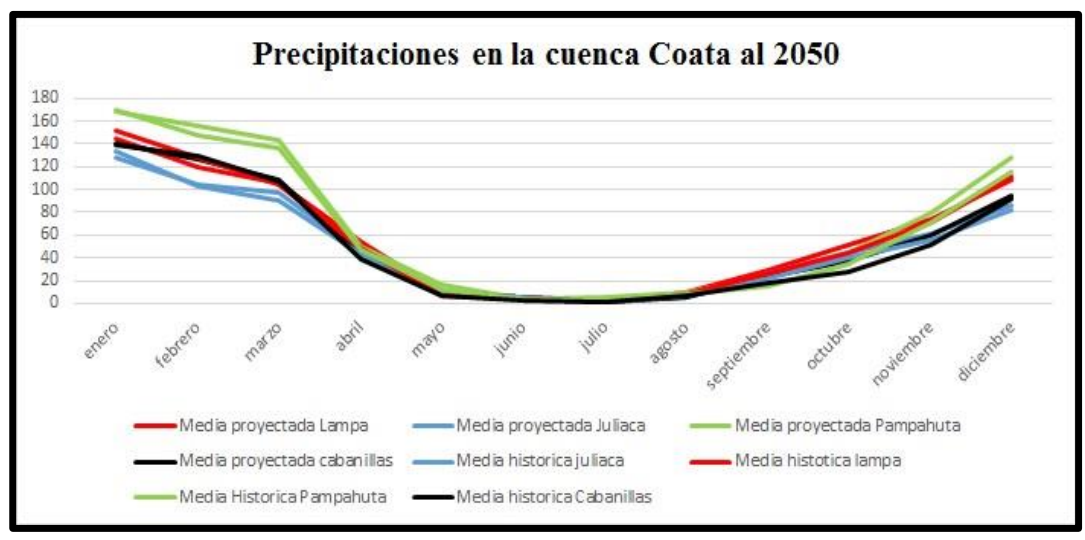

Figura 5. Variación de precipitación histórica SENAMHI Y proyectada CIMP5 
Con respecto a la precipitación menciona que se mostraron deficiencias mayormente en la zona de Lampa, también algunas estimaciones en la sierra de Puno, entre $-10 \%$ y $-20 \%$. Y en la selva norte y central con un $-10 \%$. Por tanto se concluye que en la costa norte y selva sur se darán los incrementos más importantes con un $-10 \%$ a $+20 \%$ según estudios realizados por (Lujano, et al., 2016), que paralelo a ello se estima que en la cuenca Ramis se dará un incremento de aproximadamente entre 60 a 80 centímetros para los próximos cien años.

Lo que se muestra la temperatura máxima y mínima de la Cuenca Coata proyectado al 2050.

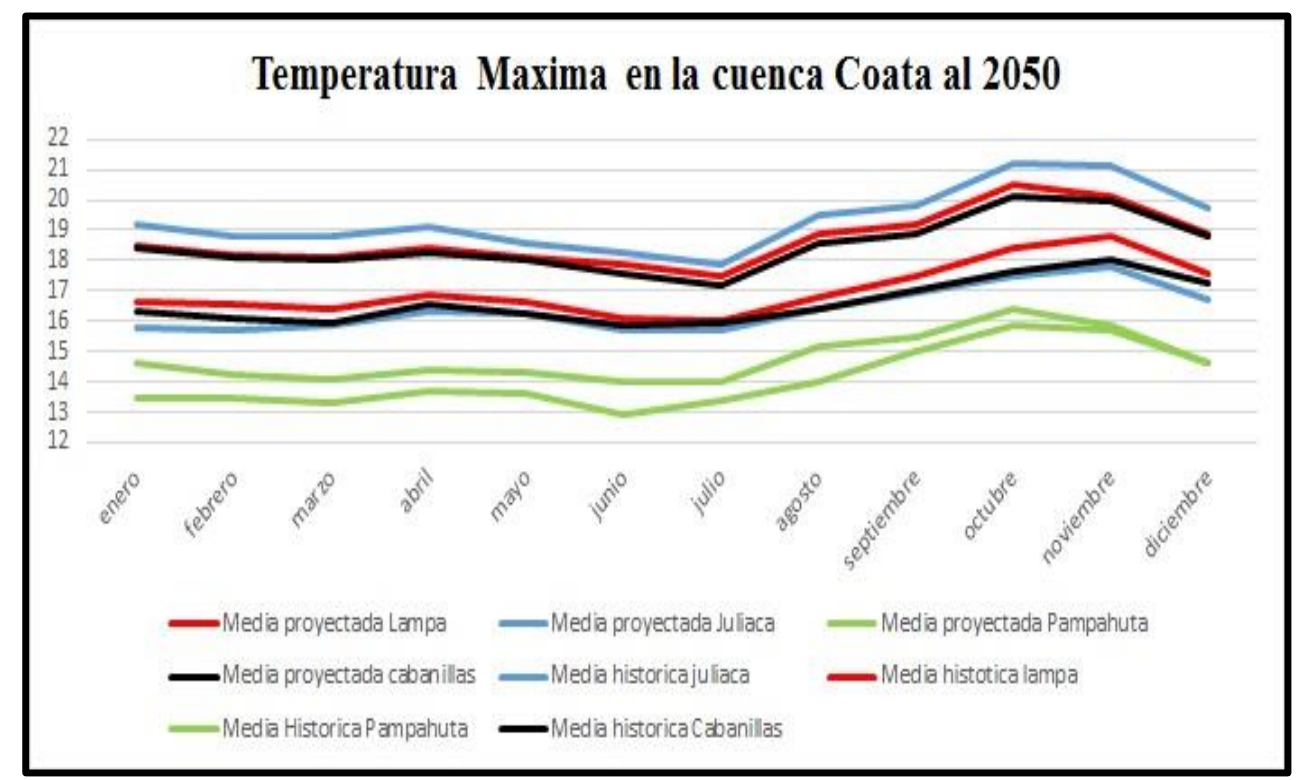

Figura. 6 Variaciones de temperaturas máximas históricas SENAMHI y proyectada CIMP5

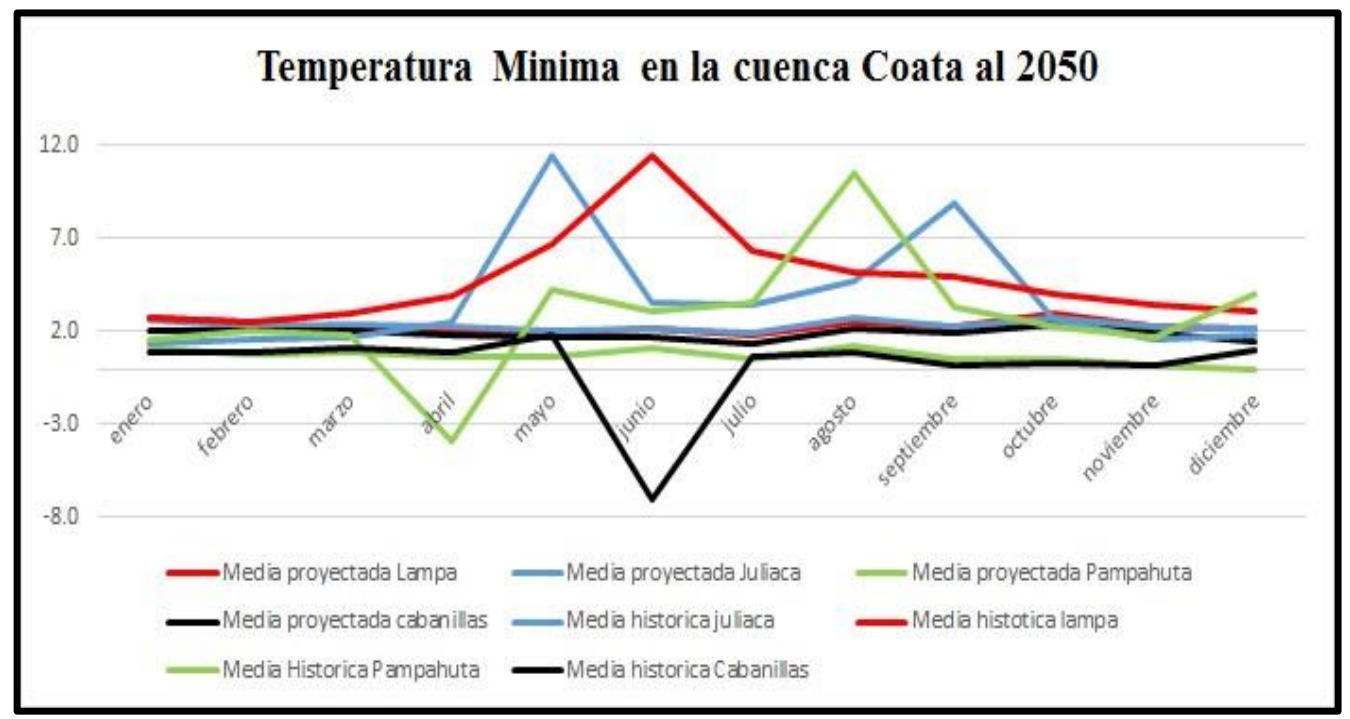

Figura. 7 Variación de temperaturas mínimas históricas SENAMHI y proyectada CIMP5 


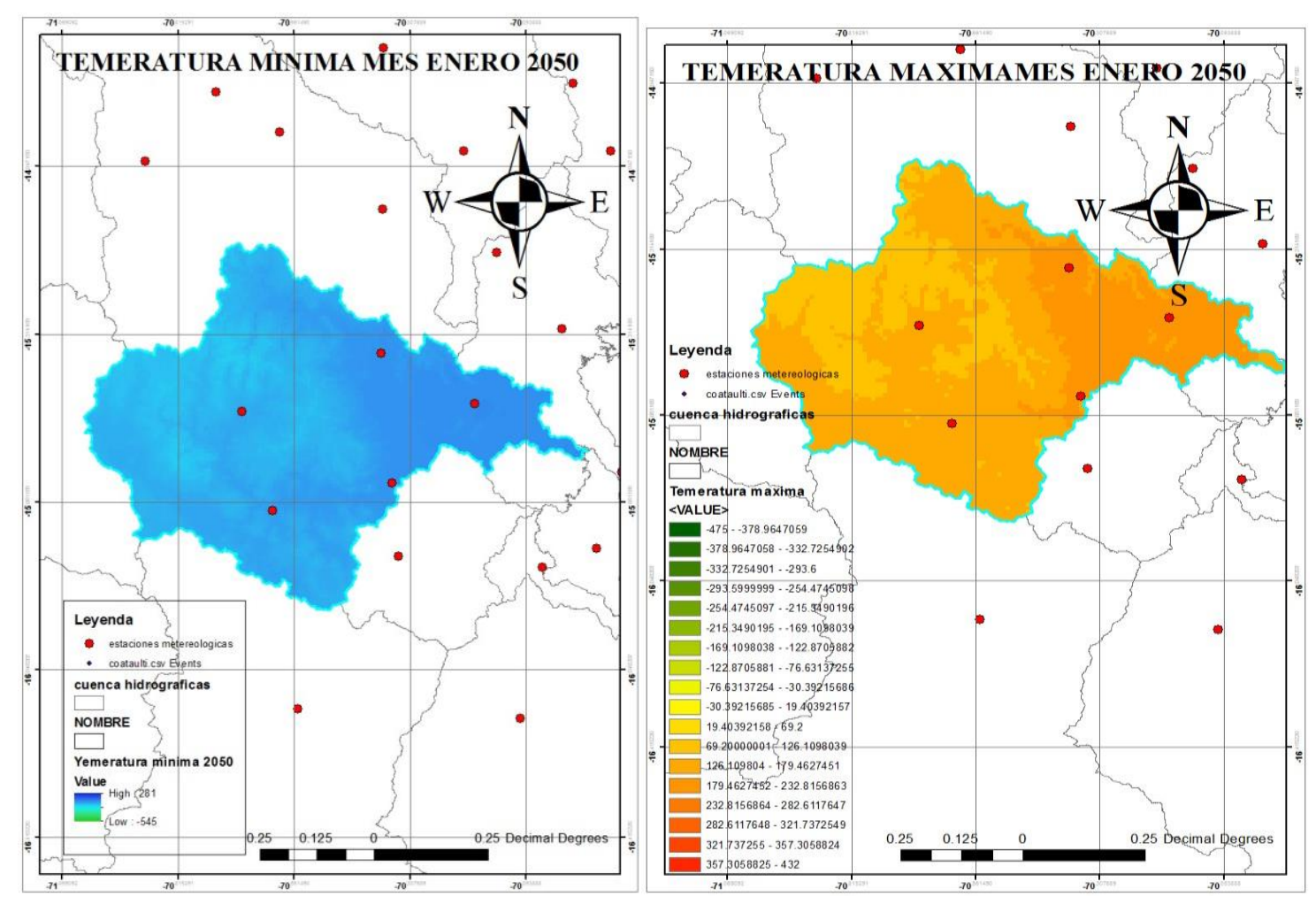

Figura. 8 Temperaturas anuales de la cuenca coata al 2050.

\section{Conclusión}

Los riesgos efectos del cambio Climático son amenazas que movilizan el interés de las familias alpaqueras por el nivel de daños y pérdidas que generan. Existe una vieja relación de las familias alpaqueras y la variabilidad climática presente en la zona, ya sea por el incremento de temperatura y los cambios en la precipitación, bajo los escenarios de cambio climático que impacta e impactará en la actualidad la distribución de especies a nivel local, regional, nacional y mundial. Si las temperaturas mínimas aumentan causa una disminución en la intensidad y frecuencia de las escarchas y consecuentemente disminuye el frío en el invierno y aumenta la sensación de calor extremo en el verano (Storino, 2009). Se verá la población en graves problemas con su producción pecuaria y agricultura.

Con respecto a la afectación de la alpaca según los resultados la temperatura va disminuir, por lo que se verá presencia de nieve y bajas temperaturas lo que cabe la posibilidad de que haya mayor cantidad de muertes de las alpacas, lo cual ante el cambio climático más extremo se deberán proteger a estas especies silvestres, evitar la exposición al friaje y es recomendable cultivar variedades de plantas adaptables a ese clima para que las alpacas no dependan solo de los pastizales, sino se adapten a las nuevas condiciones climáticas.

Esto quiere decir que para la proyección del año 2050, se ha determinado que ambos pueden verse gravemente afectados por el cambio climático y conducir a impactos estructurales, en el 
incremento de las temperaturas, por ello la posibilidad de afectación en la crianza de las alpacas, ya que en la región Puno, el frío incrementa, por ende la producción de crías.

\section{Agradecimientos}

Especial agradecimiento al Ing. Efrain Lujano, Especialista Hidrometeorológico del Servicio Nacional de Meteorología e Hidrología del Perú (SENAMHI), por su gentil apoyo en el curso de Hidrología y Manejo de Cuenca, asimismo quien prestó asesoría quien hizo posible el desarrollo del presente trabajo. Para contribuir a la investigación científica para el desarrollo Regional y del País. Asímismo, al grupo de estudio de ingeniería Ambiental; al Servicio Nacional de Meteorología e Hidrología (SENAMHI Puno por la información meteorológica.

\section{Referencias}

Andersen, J., Refsgaard, J., y Jensen, K. H. (2001). Distributed hydrological modelling of the Senegal River basin-model construction and validation. Journal Hydrological, 247, 200214.

Crispín Cunya , M. (2008). Productividad y distribucion de fibra de alpaca en la reion de Huancavelica. Lima - Perú: Universidad Mayor de San Marcos.

FAO., (2005). Situación Actual de los Camélidos Sudamericanos en el Perú. Organización de las Naciones Unidas para la Agricultura y la Alimentación. Proyecto de Cooperación Técnica en apoyo a la crianza y aprovechamiento de los Camélidos Sudamericanos en la Región Andina TCP/RLA/2914. http://www.fao.org/regiona.

INEI (2007) Avance económico y social regional. Departamento de Puno. Junio 2007. http//www1.inei.gob.pe/biblioineipub/bancopub /Est/Lib0739/index.htm

IPCC (Intergovernmental Panel on Climate Change). 2007. Climate Change 2007: Climate Change Impacts, Adaptation and Vulnerability. Summary for policy makers.IPCC WGII 4th Assessment Report.Praga, República Checa.

Lujano, E., Díaz, R. D., Lujano, A., y Quispe, J. P. (2014). Evaluación de la disponibilidad hídrica actual y futura en el contexto del cambio climático en las cuencas Ilave y Ramis. Revista de Investigaciones Altoandinas, 16(2), 65-72

Mori Kurimaya, M., \& Zafra Pozo, J. (2008). Análisis de impacto de los eventos fríos (friaje) del 2008 en la agricultura y ganadería alto andina en el Perú. Perú: Organización de las Naciones Unidas para la Agricultura y la Alimentación, FAO.

Neitzke, S. \& Alten, S. (1997). Información general sobre las alpacas. Alemania.

Sanabria J., Marengo J. y Velarde M. 2010. "Escenarios de Cambio Climático con modelos regionals sobre el Altiplano Peruano (Departamento de Puno)". Servicio Nacional de Meteorología e Hidrología, SENAMHI, Centro de Ciencias do Sistema Terrestre-INPE. Rodovia Presidente Dutra, Km 40, 12630-000 Cachoeira Paulista, São Paulo, Brasil. 
SENAMHI, (2016), Servicio Nacional de Meteorología e Hidrología. Guía Básica de Meteorología General. La tierra y su atmósfera. Perú

StorinoHolderbaum, Breno. 2009. "Cambio climático regional y turismo local”. El caso del sur de Brasil 